\title{
Quantum gates between two spins in a triple-dot system with an empty dot
}

\author{
Jose Garcia Coello* and Sougato Bose \\ Department of Physics and Astronomy, University College London, Gower St., London WC1E 6BT, United Kingdom
}

(Received 28 August 2012; published 9 July 2013)

\begin{abstract}
We propose a scheme for implementing quantum gates and entanglement between spin qubits in the outer dots of a triple-dot system with an empty central dot. The voltage applied to the central dot can be tuned to realize the gate. Our scheme exemplifies the possibility of quantum gates outside the regime where each dot has an electron, so that spin-spin exchange interaction is not the only relevant mechanism. Analytic treatment is possible by mapping the problem to a $t-J$ model. The fidelity of the entangling quantum gate between the spins is analyzed in the presence of decoherence stemming from a bath of nuclear spins, as well as from charge fluctuations. Our scheme provides an avenue for extending the scope of two-qubit gate experiments to triple dots, while requiring minimal control, namely that of the potential of a single dot, and may enhance the qubit separation to ease differential addressability.
\end{abstract}

DOI: 10.1103/PhysRevB.88.035304

PACS number(s): 03.67.Ac, 03.67.Lx

\section{INTRODUCTION}

Quantum dots (QDs) are regarded as a good system for the storage and manipulation of quantum information (QI). In these systems, the qubit could be encoded, for example, in the spin of an electron ${ }^{1-6}$ or the electronic charge distribution ${ }^{7}$ or even the presence/absence of excitons. ${ }^{8}$ Spin qubits are particularly important because of their long decoherence times. The earliest proposals advocated the use of the spin of a single electron in a quantum dot as a qubit with quantum gates being realized by tuning the tunnel coupling between two quantum dots. ${ }^{1}$ On the other hand, some early experiments ${ }^{2}$ and recent proposals ${ }^{4}$ have focused on qubits encoded on two spins in double dot systems, where the control parameter is the energy mismatch between the quantum dots. This is motivated by the fact that the energy mismatch between dots can be simple to control, for example, through source-drain bias ${ }^{9}$ or local electrostatic gates. ${ }^{2}$ It would thus be interesting to have a protocol where one requires only the above control (namely the energy mismatch between dots) and is yet able to use a single spin as a qubit. In this paper, we propose such a protocol using a linear triple-dot system where qubits (individual electronic spins) are placed in the outer dots with the central dot being kept unfilled. An alternative motivation for our work stems from the fact that various triple-dot systems are now being fabricated and their charge stability diagram with small numbers of electrons is being studied. ${ }^{10,11}$ However, most experiments in quantum information context (with the exception of Ref. 11) so far have been limited exclusively to double dot systems. It would thereby be very timely to have a scheme such as ours, which enhances the scope of quantum gate related experiments to triple-dot systems. Of course, the most straightforward generalization of the schemes in double dots ${ }^{1}$ would be to have three spin qubits in three quantum dots, i.e., the filling of the quantum dots being $(1,1,1)$. Another possibility is to have a spin in the central dot as a mediator for an effective coupling between the outer dots, a configuration which has recently been studied in the molecular context. ${ }^{12}$ Another possibility with a $(1,1,1)$ filling is to encode a single qubit in three dots, ${ }^{13}$ which has been explored in a very recent experiment. ${ }^{11}$ Here, we find out that a lower filling configuration, namely a $(1,0,1)$ filling, also provides a system for two-qubit quantum gates with the qubits being in the outer dots. Note that other instances of single qubits encoded in spin spread over several dots have been discussed before ${ }^{14}$ including the case of having empty dots to enact single qubit gates on such encoded qubits ${ }^{15}$. The $(1,0,1)$ filling considered by us prevents one from reducing the problem to one of distinguishable spins (labeled by their sites) interacting through exchange interactions as in most existing schemes for quantum gates with spin qubits. Thus a second quantized treatment is convenient in the current problem and makes an interesting approach for a clear presentation of the problem. Note that very recently an alternative mechanism for two-qubit gates in a triple-dot system with qubits in the first two dots, i.e., a $(1,1,0)$ filling, has been proposed using spin-dependent tunnelings and adiabatic processes ${ }^{16}$ - however, that is a very different scheme from the one we report here, which is neither adiabatic nor exploiting spin-dependent couplings. Moreover, fast and coherent singlet-triplet filtering mechanisms have been proposed in single dots, which effectively behave rather similarly to multiple quantum dots. ${ }^{17,18}$

\section{SETUP}

Our setup consists of three quantum dots (QDs) in a row, with the voltage applied to the central one being controllable by some electrode, as shown in Fig. 1. We label the outer dots of the chain as dot A and dot B, while we label the central dot as dot C. We will assume that the Mott-Hubbard Hamiltonian describes the system well (for example, see Ref. 19), whereby the relevant Hamiltonian is

$$
\begin{aligned}
\mathcal{H}= & \sum_{\sigma, i} E_{i} d_{i \sigma}^{\dagger} d_{i \sigma}+\sum_{\sigma} t_{\mathrm{AC}}\left(d_{\mathrm{A} \sigma}^{\dagger} d_{\mathrm{C} \sigma}+d_{\mathrm{C} \sigma}^{\dagger} d_{\mathrm{A} \sigma}\right) \\
& +\sum_{\sigma} t_{\mathrm{CB}}\left(d_{\mathrm{C} \sigma}^{\dagger} d_{\mathrm{B} \sigma}+d_{\mathrm{B} \sigma}^{\dagger} d_{\mathrm{C} \sigma}\right)+\frac{1}{2} \sum_{i} U_{i} n_{i}\left(n_{i}-1\right) .
\end{aligned}
$$

In the above, $i$ stands for $\mathrm{A}, \mathrm{B}$, and $\mathrm{C}, d_{i \sigma}^{\dagger}$ creates and $d_{i \sigma}$ annihilates an electron at the $i$ th dot in the spin state $\sigma$ with energy $E_{i}$. Here, we have assumed that the particles are created only in the lowest energy state at the site $\left(E_{i}\right)$ and the higher 


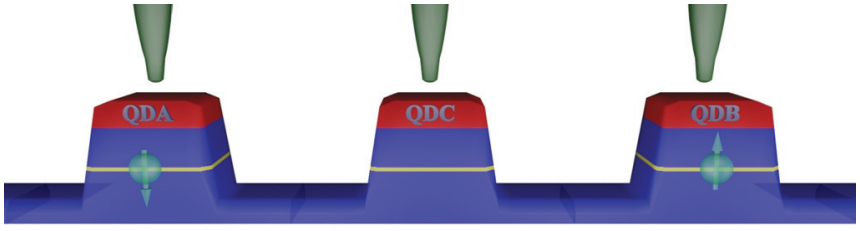

FIG. 1. (Color online) The above figure depicts the triple-dot system where we investigate the possibility of quantum gates. There are two spins in the outer dots, which behave as qubits, while the central dot is empty both before and after the quantum gates. QDA, QDC, and QDB in the figure stand for quantum dots A, C, and B, respectively, while separate electrodes controlling the voltages of each dot are also shown in the figure.

energy levels for a single electron are so well separated that they never become involved in the problem. $U_{i}$ is the Coulomb repulsion in the QD $i, n_{i}=\sum_{\sigma} d_{i \sigma}^{\dagger} d_{i \sigma}$ in the total electron number operator of the $i$ th dot and $t_{\mathrm{AC}}$ and $t_{\mathrm{CB}}$ are tunnel matrix elements between dots. Here, we have assumed that another term, often present in Hubbard models for dot arrays, namely the interdot electrostatic interaction is zero. The dots at the two ends (i.e., QD A and QD B) are each assumed to be filled up by a single electron as shown in Fig. 1. These two electronic spins will be the two qubits in our problem. As these qubits are identified by their sites, they can be referred to as qubit A and qubit B, respectively. Of course, we should be able to control when we want to enact a quantum gate between the aforementioned qubits, and for those intervals of time when we do not want any gates, nothing should happen to the qubits (the state of the qubits, whatever they are, should remain intact). To ensure this, one has to ensure that the qubits stably remain in a $(1,0,1)$ filling as shown in Fig. 1 and do not hop into QD C during this nonprocessing stage. This is achieved by choosing an appropriate set of voltages applied to the triple-dot system and there are quite a few experimental examples by now in which the $(1,0,1)$ filling has already been realized. Typically, if the Hamiltonian $\mathcal{H}$ of Eq. (1) is valid with $t_{\mathrm{AC}} \approx t_{\mathrm{CB}}=t$, then one has to set the voltage applied to $\mathrm{QD} C$ to a lower value and the voltages of QDs $\mathrm{A}$ and $\mathrm{B}$ to a higher equal value. Also, we have to work with systems with $t \ll\left|E_{\mathrm{C}}-E_{\mathrm{A}}\right|,\left|E_{\mathrm{C}}-E_{\mathrm{B}}\right|$ so that hopping is severely suppressed. In this "nonprocessing" mode of our system, the system evolution effectively freezes. When one intends to accomplish a quantum gate, one rapidly sets $E_{\mathrm{C}}=E_{\mathrm{A}}=E_{\mathrm{B}}$ and a time evolution starts (this is true as long as the Hamiltonian $\mathcal{H}$ with $t_{\mathrm{AC}} \approx t_{\mathrm{CB}}=t$ is a good approximation of the triple-dot system in consideration; in different experimental realizations, the Hamiltonian may deviate differently from this, and then, for the processing mode, one has to apply the voltage that ensures the electrostatic energy of the configurations $(1,0,1),(1,1,0)$, and $(0,1,1)$ to be equal, so that the Hamiltonian becomes $\mathcal{H}$. Thus, if we want our $\mathcal{H}$ to only act for a time duration $\tau_{0}^{\prime}$, we simply change the gate voltages fast enough at the start and end of that time duration to switch $\mathcal{H}$ on and off. We will also assume that $U_{\mathrm{A}} \approx U_{\mathrm{B}} \approx U_{\mathrm{C}}$. We will show that a two-qubit "entangling" quantum gate can be obtained between the qubits by virtue of this evolution through Hamiltonian $\mathcal{H}$. Though during the time evolution, the electrons can hop into the otherwise empty QD $\mathrm{C}$, and indeed this is necessary for their spins to interact, at the end of a fixed period of evolution, one electron is back in each of QD A and QD B. We will assume that single qubit gates on the spins in the outer dots can be trivially implemented by using local fields, so that we are going to concentrate only on the demonstration of a two-qubit entangling gate. The demonstration of the two-qubit entangling gate is at the heart of demonstrating the viability of a system for universal quantum computation.

\section{POTENTIAL PHYSICAL REALIZATIONS}

Before proceeding to demonstrating the viability of twoqubit gates, we briefly outline the potential physical systems where our scheme may be realized. Note that we have assumed that there exists no tunneling between the non-neighboring dots, namely A and B. This should be a good approximation in serial triple-dot systems ${ }^{10}$ as $A$ and $B$ have a high separation. ${ }^{10}$ Appropriate voltages for the $(1,0,1)$ filling of such triple-dot systems then have to be applied and, in fact, such voltages have already been applied in Ref. 10 to realize the $(1,0,1)$ filling. Gating each of the dots in such a triple-dot system to have either $E_{\mathrm{C}}=E_{\mathrm{A}}=E_{\mathrm{B}}$ or $t \ll\left|E_{\mathrm{C}}-E_{\mathrm{A}}\right|,\left|E_{\mathrm{C}}-E_{\mathrm{B}}\right|$ is by now quite standard, and should provide the way to switch our gates on and off as discussed above. We also need relevant experimental values for $E_{i}, U_{i}, t_{\mathrm{AC}}$, and $t_{\mathrm{CB}}$ to check the viability of our gates. We take the values of these from the tables in the experimental paper Ref. 6, which will provide our guide for exploring feasibility issues. From a different point of view, cold fermionic atoms in optical superlattices with three sites in each superlattice cell is another altogether different physical realization. ${ }^{26}$ For neutral fermionic atoms, the effects of charge noise will not be there, while tunneling can be controlled by changing the well depths.

\section{THE TWO QUBIT GATE}

The specific gate that we will demonstrate as enactable between the spins in the outer dots by means of their evolution through the Hamiltonian $\mathcal{H}$ is given by the following evolution of the computational basis states $|\uparrow\rangle$ (up spin along any axis, say $z$, standing for the logical state $|0\rangle$ ) and $|\downarrow\rangle$ (down spin along any axis, say $z$ standing for the logical state $|1\rangle)$ :

$$
\begin{aligned}
|\uparrow\rangle_{A}|\uparrow\rangle_{B} & \rightarrow|\uparrow\rangle_{A}|\uparrow\rangle_{B}, \\
|\uparrow\rangle_{A}|\downarrow\rangle_{B} & \rightarrow e^{i \frac{\pi}{4}} \frac{1}{\sqrt{2}}\left(|\uparrow\rangle_{A}|\downarrow\rangle_{B}-i|\downarrow\rangle_{A}|\uparrow\rangle_{B}\right), \\
|\downarrow\rangle_{A}|\uparrow\rangle_{B} & \rightarrow e^{i \frac{\pi}{4}} \frac{1}{\sqrt{2}}\left(|\downarrow\rangle_{A}|\uparrow\rangle_{B}-i|\uparrow\rangle_{A}|\downarrow\rangle_{B}\right), \\
|\downarrow\rangle_{A}|\downarrow\rangle_{B} & \rightarrow|\downarrow\rangle_{A}|\downarrow\rangle_{B} .
\end{aligned}
$$

Note that the above gate is manifestly an entangling quantum gate as it takes the initial states $|\uparrow\rangle_{A}|\downarrow\rangle_{B}$ and $|\downarrow\rangle_{A}|\uparrow\rangle_{B}$ to entangled states. Thus the above gate suffices, in conjunction with local unitary operations on qubits $\mathrm{A}$ and $\mathrm{B}$, for universal quantum computation. ${ }^{20}$ This gate has been called the $\sqrt{\text { SWAP }}$ gate by Loss and DiVincenzo. ${ }^{1}$

Before proceeding further, we have to briefly clarify the notations that we will use. The gate presented above is in the usual notation of states of multiple qubits, where all the qubits are distinguishable and each qubit has its own distinct 
label. However, this distinctive labels (namely, qubit A and qubit B) are true only in the "nonprocessing" phase, i.e., before and after the time evolution by $H$. The two electrons may loose their site labels (namely A and B) during the evolution and thereby a fully second quantized treatment, which automatically takes account of the indistinguishability of the electrons, is convenient. So, as basis states for writing down the Hamiltonian of the system, we shall use the states $d_{i \sigma}^{\dagger} d_{j \sigma^{\prime}}^{\dagger}|0\rangle$ with $i, j=\mathrm{A}, \mathrm{B}, \mathrm{C}$ and $\sigma, \sigma^{\prime}=\uparrow, \downarrow$, where $|0\rangle$ is the state with all three dots empty, and evaluate the matrix elements of the Hamiltonian $H$ in this basis.

Let us point out that the total spin component along any axis is conserved by $H$. Choosing an axis to be the $z$ axis, for example, and remembering our $(1,0,1)$ initial filling, the problem becomes three independent problems for the total $z$ component of the spin in the three sites $S_{z}$ being +1 $\left(\sum_{i} d_{i \uparrow}^{\dagger} d_{i \uparrow}=2\right), 0\left(\sum_{i} d_{i \uparrow}^{\dagger} d_{i \uparrow}=1\right)$ or $-1\left(\sum_{i} d_{i \uparrow}^{\dagger} d_{i \uparrow}=0\right)$. In the $S_{z}=+1$ sector, a complete basis comprises three states $d_{\mathrm{A} \uparrow}^{\dagger} d_{\mathrm{C} \uparrow}^{\dagger}|0\rangle, d_{\mathrm{A} \uparrow}^{\dagger} d_{\mathrm{B} \uparrow}^{\dagger}|0\rangle$ and $d_{\mathrm{C} \uparrow}^{\dagger} d_{\mathrm{B} \uparrow}^{\dagger}|0\rangle$, in which the $3 \times 3$ Hamiltonian is simply

$$
H_{S_{z}=+1}=\left(\begin{array}{ccc}
0 & t & t \\
t & 0 & 0 \\
t & 0 & 0
\end{array}\right) .
$$

From the above Hamiltonian, it is easy to see that if the system starts in the two-qubit state $|\uparrow\rangle_{A}|\uparrow\rangle_{B}$ (which actually means the state $\left.d_{\mathrm{A} \uparrow}^{\dagger} d_{\mathrm{B} \uparrow}^{\dagger}|0\rangle\right)$, then at times $\tau_{m}=m \frac{2 \pi}{\sqrt{2} t}$, where $m$ is an integer, the system comes back to its original state without any phase factor. Thereby, if we halt the evolution at any of these instances of time (by suddenly setting the voltages to the nonprocessing mode), we will have the $|\uparrow\rangle_{A}|\uparrow\rangle_{B} \rightarrow|\uparrow\rangle_{A}|\uparrow\rangle_{B}$ part of the quantum gate in Eq. (2) satisfied. Exactly the same result holds for the $|\downarrow\rangle_{A}|\downarrow\rangle_{B} \rightarrow|\downarrow\rangle_{A}|\downarrow\rangle_{B}$ part of the quantum gate, which evolves in the $S_{z}=-1$ sector with an identical Hamiltonian matrix. Therefore it remains to check whether there exist any values of $m$ for which the remainder of the quantum gate of Eq. (2) happens at $\tau_{m}$. For that, we have to look at the Hamiltonian in the $S_{z}=0$ sector.

\section{THE EVOLUTION IN THE $S_{z}=0$ SECTOR AND DEMONSTRATION OF THE GATE}

In the $S_{z}=0$ sector, a complete basis is made of the nine states $d_{\mathrm{A} \uparrow}^{\dagger} d_{\mathrm{C} \downarrow}^{\dagger}|0\rangle, d_{\mathrm{A} \downarrow}^{\dagger} d_{\mathrm{C} \uparrow}^{\dagger}|0\rangle, d_{\mathrm{C} \uparrow}^{\dagger} d_{\mathrm{B} \downarrow}^{\dagger}|0\rangle, d_{\mathrm{C} \downarrow}^{\dagger} d_{\mathrm{B} \uparrow}^{\dagger}|0\rangle$, $d_{\mathrm{A} \uparrow}^{\dagger} d_{\mathrm{B} \downarrow}^{\dagger}|0\rangle, d_{\mathrm{A} \downarrow}^{\dagger} d_{\mathrm{B} \uparrow}^{\dagger}|0\rangle, d_{\mathrm{A} \uparrow}^{\dagger} d_{\mathrm{A} \downarrow}^{\dagger}|0\rangle, d_{\mathrm{B} \uparrow}^{\dagger} d_{\mathrm{B} \downarrow}^{\dagger}|0\rangle, d_{\mathrm{C} \uparrow}^{\dagger} d_{\mathrm{C} \downarrow}^{\dagger}|0\rangle$. The $9 \times 9$ Hamiltonian matrix in this basis is not reproduced here for brevity, but it is important to note that here some elements such as $\left\langle 0\left|d_{\mathrm{A} \uparrow} d_{\mathrm{C} \downarrow} \mathcal{H} d_{\mathrm{A} \uparrow}^{\dagger} d_{\mathrm{A} \downarrow}^{\dagger}\right| 0\right\rangle$ are $t$, while others such as $\left\langle 0\left|d_{\mathrm{A} \downarrow} d_{\mathrm{C} \uparrow} \mathcal{H} d_{\mathrm{A} \uparrow}^{\dagger} d_{\mathrm{A} \downarrow}^{\dagger}\right| 0\right\rangle$ are $-t$. This sign difference is important. Now assuming $U>>t$, one can adiabatically eliminate the double occupancy states $d_{\mathrm{A} \uparrow}^{\dagger} d_{\mathrm{A} \downarrow}^{\dagger}|0\rangle, d_{\mathrm{B} \uparrow}^{\dagger} d_{\mathrm{B} \downarrow}^{\dagger}|0\rangle, d_{\mathrm{C} \uparrow}^{\dagger} d_{\mathrm{C} \downarrow}^{\dagger}|0\rangle$ to obtain the effective Hamiltonian

$$
\mathcal{H}_{\text {eff }}=\left(\begin{array}{rrrrrr}
-2 J & 2 J & -J & J & t & 0 \\
2 J & -2 J & J & -J & 0 & t \\
-J & J & -2 J & 2 J & t & 0 \\
J & -J & 2 J & -2 J & 0 & t \\
t & 0 & t & 0 & 0 & 0 \\
0 & t & 0 & t & 0 & 0
\end{array}\right)
$$

with $J=t^{2} / U$. The above effective Hamiltonian is that of a three-site $t-J$ model, with a parameter $t$ for hopping and a parameter $J$ for the spin-spin interaction only when the spins are in neighboring sites. We define $\eta^{ \pm}=-\left(3 J \pm \sqrt{9 J^{2}+2 t^{2}}\right)$ and $\xi^{ \pm}=\sqrt{2+\left(\eta^{ \pm}\right)^{2} / t^{2}}$, in terms of which, the eigenvalues of $H_{\text {eff }}$ are $\left\{0,-2 J,-\sqrt{2} t, \sqrt{2} t, \eta^{+}, \eta^{-}\right\}$, while its eigenvectors are

$$
\begin{aligned}
& \left|v_{1}\right\rangle=\left\{\frac{1}{2}, \frac{1}{2},-\frac{1}{2},-\frac{1}{2}, 0,0\right\}, \\
& \left|v_{2}\right\rangle=\left\{-\frac{1}{2}, \frac{1}{2}, \frac{1}{2},-\frac{1}{2}, 0,0\right\}, \\
& \left|v_{3}\right\rangle=\left\{-\frac{1}{2 \sqrt{2}},-\frac{1}{2 \sqrt{2}},-\frac{1}{2 \sqrt{2}},-\frac{1}{2 \sqrt{2}}, \frac{1}{2}, \frac{1}{2}\right\}, \\
& \left|v_{4}\right\rangle=\left\{\frac{1}{2 \sqrt{2}}, \frac{1}{2 \sqrt{2}}, \frac{1}{2 \sqrt{2}}, \frac{1}{2 \sqrt{2}}, \frac{1}{2}, \frac{1}{2}\right\}, \\
& \left|v_{5}\right\rangle=\left\{\frac{\eta^{+}}{2 t \xi^{+}},-\frac{\eta^{+}}{2 t \xi^{+}}, \frac{\eta^{+}}{2 t \xi^{+}},-\frac{\eta^{+}}{2 t \xi^{+}},-\frac{1}{\xi^{+}},+\frac{1}{\xi^{+}}\right\}, \\
& \left|v_{6}\right\rangle=\left\{\frac{\eta^{-}}{2 t \xi^{-}},-\frac{\eta^{-}}{2 t \xi^{-}}, \frac{\eta^{-}}{2 t \xi^{-}},-\frac{\eta^{-}}{2 t \xi^{-}}, \frac{1}{\xi^{-}},-\frac{1}{\xi^{-}}\right\} .
\end{aligned}
$$

We want to show that the initial state $|\uparrow\rangle_{A}|\downarrow\rangle_{B}$ of qubits A and B evolves to

$$
\left|\psi_{\text {target }, \uparrow \downarrow}\right\rangle=e^{i \pi / 4} \frac{1}{\sqrt{2}}\left(|\uparrow\rangle_{A}|\downarrow\rangle_{B}-i|\downarrow\rangle_{A}|\uparrow\rangle_{B}\right)
$$

at a certain time under the action of the Hamiltonian $\mathcal{H}_{\text {eff }}$. Moreover, this time must be coincident or approximately coincident with $\tau_{m}=m \frac{2 \pi}{\sqrt{2} t}$ (discussed in the previous section) for some $m$, so that the gate of Eq. (2) is accomplished at the time $\tau_{m}$. The initial state $|\uparrow\rangle_{A}|\downarrow\rangle_{B}$, or more accurately the second quantized state $d_{\mathrm{A} \uparrow}^{\dagger} d_{\mathrm{B} \downarrow}^{\dagger}|0\rangle$, evolves with time $\tau$ as

$$
\begin{aligned}
\left|\psi_{A \uparrow, B \downarrow}(\tau)\right\rangle= & \frac{1}{2}\left\{e^{i \sqrt{2} t \tau}\left|v_{3}\right\rangle+e^{-i \sqrt{2} t \tau}\left|v_{4}\right\rangle\right\} \\
& -\frac{e^{-i \eta^{+} \tau}}{\xi^{+}}\left|v_{5}\right\rangle+\frac{e^{-i \eta^{-} \tau}}{\xi^{-}}\left|v_{6}\right\rangle .
\end{aligned}
$$

If we now once more invoke $U \gg t$ to neglect terms of $O(t / U)$, we can simplify the modulus squared overlap $\left|\left\langle\psi_{A \uparrow, B \downarrow}(\tau) \mid \psi_{\text {target, } \uparrow \downarrow}\right\rangle\right|^{2}$ to the analytic expression

$$
\begin{aligned}
& \left|\left\langle\psi_{A \uparrow, B \downarrow}(\tau) \mid \psi_{\text {target }, \uparrow \downarrow}\right\rangle\right|^{2} \\
& =\frac{\cos ^{2} \sqrt{2} t \tau}{8}\left\{\left[1+\sqrt{2} \cos \left(3 J \tau-\frac{\pi}{4}\right)\right]^{2}\right. \\
& \left.\quad+\left[1-\sqrt{2} \cos \left(3 J \tau+\frac{\pi}{4}\right)\right]^{2}\right\} .
\end{aligned}
$$

We will see later on, comparing with the numerical plots, that this expression is valid to a high accuracy for $U=200 \mu \mathrm{eV}$ and $t=\sqrt{200} \mu \mathrm{eV}$ and even better for higher $U / t$ ratios. Notice that there are two distinct frequencies in the above expression, namely the higher frequency $\sqrt{2} t$, which is due to the tunneling, and the much lower frequency $3 J$, which is due to the spin-spin interactions. Also note that, as expected, the modulus squared overlap with the target state is 0.5 at time $\tau=0$. However, most important to note is that at times $\tau_{n}^{\prime}=(2 n+1) \pi / 6 J$ with $n$ being an integer, the modulus squared overlap is unity implying that at these instances, the 


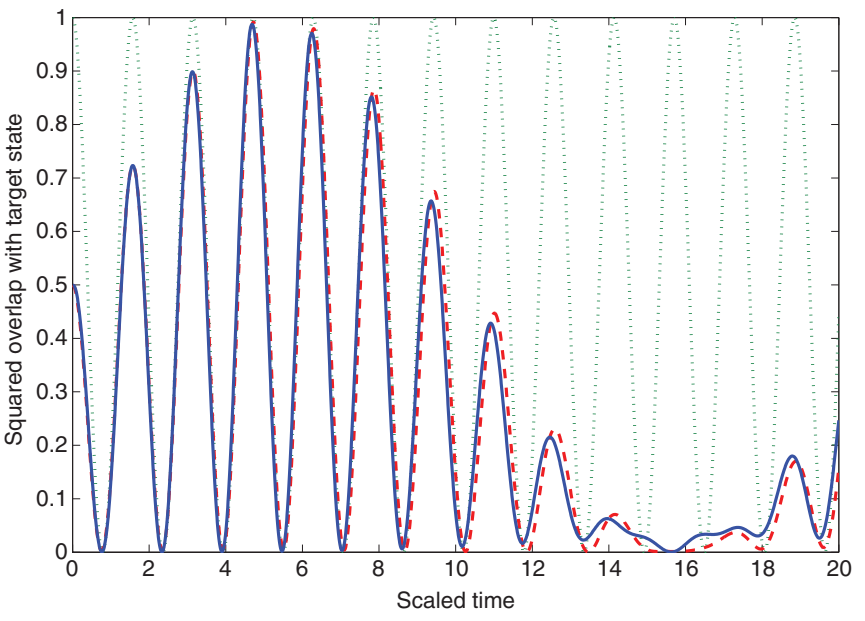

FIG. 2. (Color online) Plots to demonstrate the occurrence of an entangling quantum gate at a certain instant of time between the spins $\mathrm{A}$ and $\mathrm{B}$. The dotted line is the modulus squared overlap of $|\uparrow\rangle_{A}|\uparrow\rangle_{B}$ with the state it evolves to as a function of time after the gating Hamiltonian is switched on. Both the solid and the dashed lines show the modulus squared overlap of $\frac{1}{\sqrt{2}}\left(|\uparrow\rangle_{A}|\downarrow\rangle_{B}-i|\downarrow\rangle_{A}|\uparrow\rangle_{B}\right)$ with the state to which $|\uparrow\rangle_{A}|\downarrow\rangle_{B}$ evolves as a function of time after the gating Hamiltonian is switched on. The solid line is from our analytic expression of Eq. (8), while the dashed line from numerics without approximations. The parameters used in the plot are $t=\sqrt{2}$ and $U=20$ in scaled units where the energy scale $10 \mu \mathrm{eV}$ is set to unity (one unit of the scaled time is about $0.45 \mathrm{~ns}$ ).

initial state $|\uparrow\rangle_{A}|\downarrow\rangle_{B}$ of qubits A and B has fully evolved to the entangled state $e^{i \frac{\pi}{4}} \frac{1}{\sqrt{2}}\left(|\uparrow\rangle_{A}|\downarrow\rangle_{B}-i|\downarrow\rangle_{A}|\uparrow\rangle_{B}\right)$. By following identical steps as above, one can prove that at times $\tau_{n}^{\prime}$ the initial state $|\downarrow\rangle_{A}|\uparrow\rangle_{B}$ of qubits $\mathrm{A}$ and $\mathrm{B}$ evolves to $e^{i \frac{\pi}{4}} \frac{1}{\sqrt{2}}\left(|\downarrow\rangle_{A}|\uparrow\rangle_{B}-i|\uparrow\rangle_{A}|\downarrow\rangle_{B}\right)$. As $2 \pi / \sqrt{2} t \ll \pi / 6 J$, for any $\tau_{n}^{\prime}$ there will exist several values of $m$ for which $\tau_{m}$ is close to $\tau_{n}^{\prime}$. Thus one can always choose some $m$ and $n$ so that $\tau_{m} \approx \tau_{n}^{\prime}$ and at this particular time the quantum gate of Eq. (2) is accomplished. Ideally, we would like to choose the shortest possible time to accomplish the quantum gate to minimize the effects of decoherence. The earliest opportunity is at time $\tau_{0}^{\prime}$ as this is the earliest time the second and third lines of the gate of Eq. (2) are accomplished. Depending on the strength of the tunnel coupling $t$, nearly always it is possible to find an $m$ such that $\tau_{m} \approx \tau_{0}^{\prime}$ so that the quantum gate of Eq. (2) is accomplished at $\tau_{0}^{\prime}$. To convince the readers about this, we take explicit values of parameters in scaled units. First, we set the energy scale of about $10 \mu \mathrm{eV}$, which is a realistic typical scale of $t^{6,21,22}$ to unity. In these units, we take $t=\sqrt{2}$ and $U=20$ so that $U>>t$ is valid and yet $J \sim 0.1$ is not too small. Such ratios of $U / t$ are available realistically ${ }^{21,22}$ and we plot some relevant curves in Fig. 2.

It is clear from the figure that the modulus squared overlaps of the $|\uparrow\rangle_{A}|\uparrow\rangle_{B}$ state with itself and the $|\uparrow\rangle_{A}|\downarrow\rangle_{B}$ with $\frac{1}{\sqrt{2}}\left(|\uparrow\rangle_{A}|\downarrow\rangle_{B}-i|\downarrow\rangle_{A}|\uparrow\rangle_{B}\right)$, both achieve values indistinguishable from unity at time $\tau_{0}^{\prime}$. Further, note that one could always tune the two free parameters $t$ and $U$, to ensure that $\tau_{0}^{\prime} \approx \tau_{m}$ holds for some $m$. Figure 2 also presents a plot for the evolution of $|\uparrow\rangle_{A}|\downarrow\rangle_{B}$ to $\frac{1}{\sqrt{2}}\left(|\uparrow\rangle_{A}|\downarrow\rangle_{B}-i|\downarrow\rangle_{A}|\uparrow\rangle_{B}\right)$ from exact numerical diagonalization of Eq. (1) to show that the approximations (adiabatic elimination) leading to the expression of Eq. (8) are valid. However, to verify the quantum gate, one also needs to verify the phases outside the brackets on the right-hand sides of the second and third lines of Eq. (2). We temporarily postpone this, and will verify these through additional plots that we make in the next section where we treat decoherence.

Note that we have used only the commutation of $H$ with $S_{z}$ in this section to simplify our problem and not used the full symmetry of the Hamiltonian as $H$ also commutes with $S^{2}=S_{x}^{2}+S_{y}^{2}+S_{z}^{2}$. As it happened, it was sufficient for us to split the $H$ matrix to simpler sectors using its commutation with $S_{z}$. The greater symmetry, though, will enable one to explain the physical origin of the $\sqrt{\text { SWAP }}$ gate of Eq. (2). Note that an initial triplet state of the spins in the dots $A$ and $B$ does not acquire any phase whereas the singlet state acquires a phase of $e^{i \pi / 2}$ as a result of the gate. This is because two spins in a triplet spin state will have an antisymmetric spatial wave function, and consequently, after one of them hops to the site $C$, the electrons will not be able to go through the double occupancies $[(2,0,0),(0,2,0)$, or $(0,0,2)]$ needed for a spin exchange interaction between $C$ and $A$ or $C$ and $B$.

\section{ROLE OF NOISE AND DECOHERENCE}

Now that we have demonstrated the possibility of an entangling gate between the spin qubits in our triple-dot setting, we proceed to investigate how this gate is affected by various sources of decoherence. During the fleetingly small time window of gate operation (about a nanosecond) transient charge superpositions will exist, and thereby the gate will be subject to some charge decoherence despite operating between spin qubits. Note that this is not unique to our setting, but, in fact, also automatically present when one intends to implement two-qubit gates with singlet-triplet qubits defined in double dots. There, the singlet and the triplet have to go to distinct charge configurations to enable gates between two double-dot qubits. ${ }^{4}$ As such decoherence is only during the gate operation, one can suppress it effectively by making the gate faster (i.e., $J$ stronger). In our case, during storage of the qubits, though, only spin decoherence, primarily due to the hyperfine interaction with nuclear spins, will be present.

We first model the effect of charge decoherence numerically. As the temperature is lowered enough so that the effect of phonons is eliminated (this assumption is met in current quantum dot experiments), decoherence due to spin-orbit interactions is suppressed. The $1 / f$ noise generated in the triple-dot device due to the fluctuations in the background charge is then the predominant source of decoherence. We will phenomenologically fix the amplitude of this noise to set a charge decoherence time scale of about $1 \mathrm{~ns}$ (coherent charge oscillations have been observed till about $2 \mathrm{~ns}^{23}$ and even much higher have been reported in nongated devices. ${ }^{24}$ ) Setting the amplitude in this phenomenological way also has the advantage that it models charge decoherence of the best observed strengths irrespective of its cause (for example, some phonons may still be present). We have numerically generated 


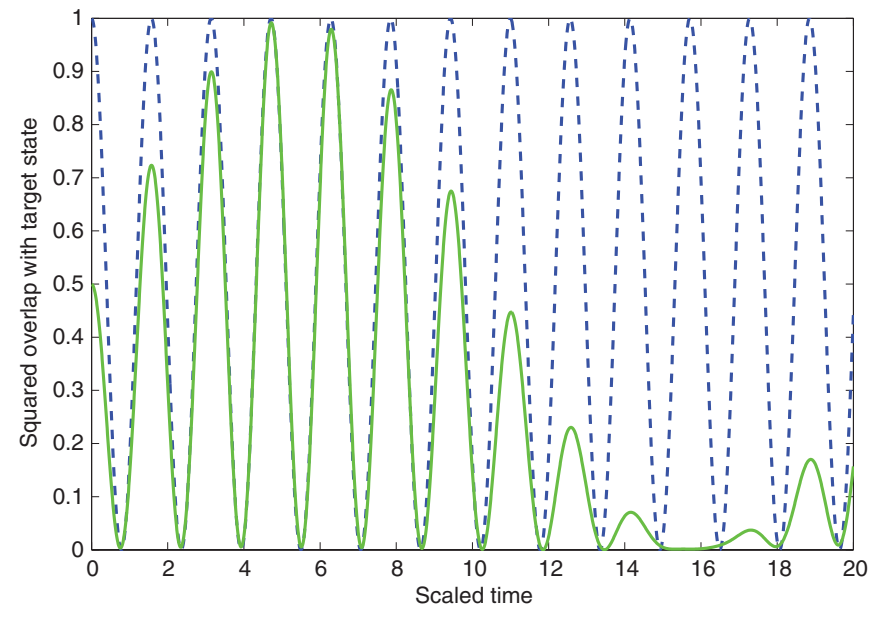

FIG. 3. (Color online) The figure shows the effect of charge decoherence on the quantum gate of our protocol. We induce a charge decoherence time scale of about 1 ns (about 10 units of our scaled time) by appropriately tuning a $1 / f$ noise. The time evolution of the modulus squared overlap of an initial $|\uparrow\rangle_{A}|\uparrow\rangle_{B}$ state under this noise with itself (dashed curve) shows the purely charge based decoherence effect. Keeping the parameters of the charge noise the same, we have also plotted the modulus squared overlap of the state $\frac{1}{\sqrt{2}}\left(|\uparrow\rangle_{A}|\downarrow\rangle_{B}-i|\downarrow\rangle_{A}|\uparrow\rangle_{B}\right)$ with the state to which $|\uparrow\rangle_{A}|\downarrow\rangle_{B}$ evolves as a function of time after the gating Hamiltonian is switched on (solid curve). The parameters used in the plot are $t=\sqrt{2}$ and $U=20$ in scaled units where the energy scale $10 \mu \mathrm{eV}$ is set to unity (one unit of the scaled time is about $0.45 \mathrm{~ns}$.)

a $1 / f$ noise and used a distinct value of the noise in each time step. The numerical program that generates the noise guarantees that it has $1 / f$ noise spectrum. We have also taken the tunneling $t$ to change with the mismatch of the dot energies-we have taken $t$ to vary with the energy mismatch with a narrow Gaussian profile of width 0.01 (this profile of $t$ has been taken only for this phenomenological decoherence estimation and not elsewhere in the paper). We then vary the average strength of the fluctuations till we get about a nanosecond time scale of decay of the oscillations of the state $|\uparrow\rangle_{A}|\uparrow\rangle_{B}$ during the gate, which are essentially purely charge oscillations. This is plotted in Fig. 3. We now take the same strength of noise for the evolution of $|\uparrow\rangle_{A}|\downarrow\rangle_{B}$ under the gate and numerically plot (in Fig. 3) the probability of it to evolve to its ideal target state $e^{i \frac{\pi}{4}} \frac{1}{\sqrt{2}}\left(|\uparrow\rangle_{A}|\downarrow\rangle_{B}-i|\downarrow\rangle_{A}|\uparrow\rangle_{B}\right)$. From the plot, one can see that the effect of charge decoherence is not significant (the probability of the gate driving the initial states to their right targets is higher than 0.95 for both states). This has happened because we have chosen parameters carefully enough to get a $J$, which can give a gate faster than the currently known charge decoherence rates.

An additional form of decoherence that will be active is the nuclear baths in the quantum dots, which induce decoherence of the spin states. It is known that the orientations of the nuclear spins evolve at a much slower time scale in comparison to the dynamics of the electrons (time-scales of $1 / t$ and $1 / J$ ) in quantum dot systems ${ }^{6}$ so that during one operation of our gate we may effectively regard the nuclear bath to provide a random but fixed (frozen in time) field. This is known as the quasistatic approximation. ${ }^{6}$ The effect

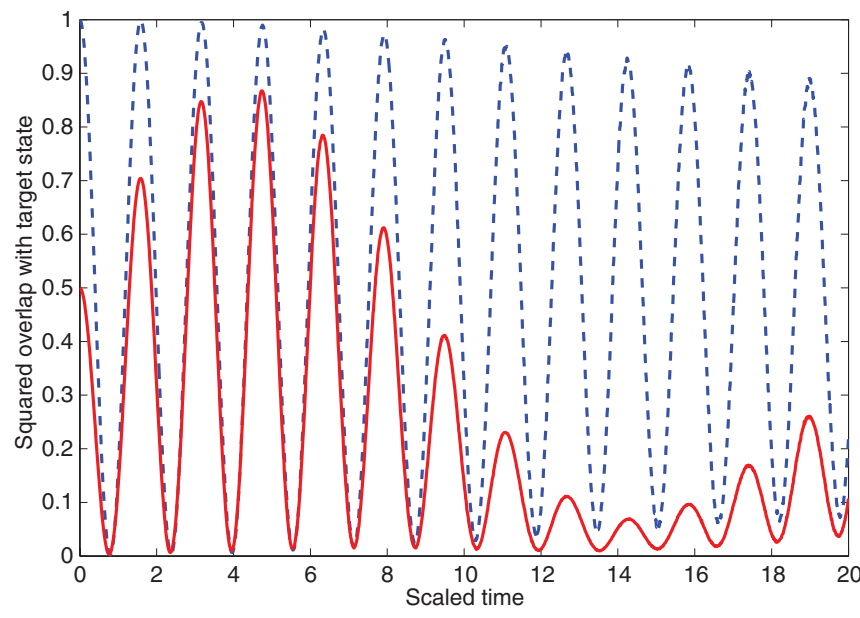

FIG. 4. (Color online) This plot shows the combined effect of both hyperfine interactions and charge decoherence on the quantum gate proposed by us. Charge noise is set so as to have a charge decoherence time scale of about $1 \mathrm{~ns}$, while the strength of the random nuclear field causing the spin decoherence is set to the realistic value of $B_{\text {nuc }} \sim 0.1$ in scaled units (with $10 \mu \mathrm{eV}$ taken as unity). The time evolution of the modulus squared overlap of an initial $|\uparrow\rangle_{A}|\uparrow\rangle_{B}$ state under this noise with itself is shown as the dashed curve, while the modulus squared overlap of the state $\frac{1}{\sqrt{2}}\left(|\uparrow\rangle_{A}|\downarrow\rangle_{B}-i|\downarrow\rangle_{A}|\uparrow\rangle_{B}\right)$ with the state to which $|\uparrow\rangle_{A}|\downarrow\rangle_{B}$ evolves as a function of time after the gating Hamiltonian is switched on is shown as the solid curve. The parameters used in the plot are $t=\sqrt{2}$ and $U=20$ in scaled units where the energy scale $10 \mu \mathrm{eV}$ is set to unity (one unit of the scaled time is about $0.45 \mathrm{~ns}$ ).

of decoherence is then due to different constant fields in various runs of the gate (a distinct random direction and magnitude in each of the quantum dots for each run of the gate). Following the parameters given in Ref. 6, we have modeled the dynamics using a magnetic field of about an order of magnitude less than the tunneling $t$ in a random direction. The direction is chosen completely at random, while the magnitude is chosen from a Gaussian distribution given as $P(B)=$ $\frac{1}{\left(2 \pi B_{\mathrm{nuc}}^{2}\right)^{3 / 2}} \exp \left(-B^{2} / 2 B_{\mathrm{nuc}}^{2}\right)$, where $B_{\mathrm{nuc}}$ is the root mean square magnetic field of the collection of all the nuclear spins in the dot. Here, one cannot really use restricted spaces any more and the full Hilbert space of the problem is involved as the nuclear magnetic field connects these spaces. Thereby we tackle this part of the problem numerically in the full Hilbert space consisting of the $S_{z}=0, \pm 1$ sectors by exact diagonalization of $\mathcal{H}$ with the addition of a random magnetic field term in each dot and using a charge decoherence of the same strength as before. The results are plotted in Fig. 4 and show that the probability of successful occurrence of the quantum gate [see Eq. (2)] remains higher than 0.9 for $B_{\text {nuc }} \sim 0.1$ in our units, which is comparable to its experimental values. ${ }^{6}$ In principle, though, this decoherence can be eliminated to a large degree by polarizing the background nuclear spins ${ }^{25}$ so that one can have quantum gates with fidelity only restricted by charge decoherence in a fleetingly small time window of gate operation. Even this latter decoherence should decrease with technology, and have already been reported to have very low values in nongated devices. ${ }^{24}$ Alternatively, it is known that quantum dotlike experiments can be performed also with 
neutral fermionic atoms in optical lattices ${ }^{26}$ where charge decoherence is inactive.

Now we return to the issue of verifying all features of the gate of Eq. (2) through appropriate plots. To verify all features of a quantum gate, one really (ideally) needs to find the closeness of the completely positive map realized in presence of decoherence with the unitary operation corresponding to the gate, i.e., the gate fidelity. However, we are going to use, for simplicity, a poor man's way of verifying the gate, which we think suffices as a witness of the reliability of the gate. Once the fidelity of the computational basis states going to the ideal ones are verified, what is left to verify is the error in the relative phases between the computational basis states due to the decoherence. We thus need to verify that the phases outside the second and third lines of the right-hand side of Eq. (2), and particularly, how it gets affected by decoherence. One way to examine this is to use $\frac{1}{\sqrt{2}}\left(|\uparrow\rangle_{A}|\uparrow\rangle_{B}+|\uparrow\rangle_{A}|\downarrow\rangle_{B}\right)$ as an initial state and verify how close it evolves to the ideal state (i.e., state under no decoherence) $\frac{1}{\sqrt{2}}|\uparrow\rangle_{A}|\uparrow\rangle_{B}+\frac{e^{i \pi / 4}}{2}\left(|\uparrow\rangle_{A}|\downarrow\rangle_{B}-\right.$ $i|\downarrow\rangle_{A}|\uparrow\rangle_{B}$ ) at time $\tau_{0}^{\prime}$. This is demonstrated under only charge decoherence and both charge and hyperfine interaction induced decoherences in Fig. 5. This shows the reliability of the gate because it examines whether a superposition of input states to the gate, evolves to the correct superposition of output states.

Before concluding this section, we would like to point out that we have assumed a fine tuning of parameters in this paper so that $t_{\mathrm{AC}} \approx t_{\mathrm{CB}}=t$ and $U_{\mathrm{A}} \approx U_{\mathrm{B}} \approx U_{\mathrm{C}}=U$. We

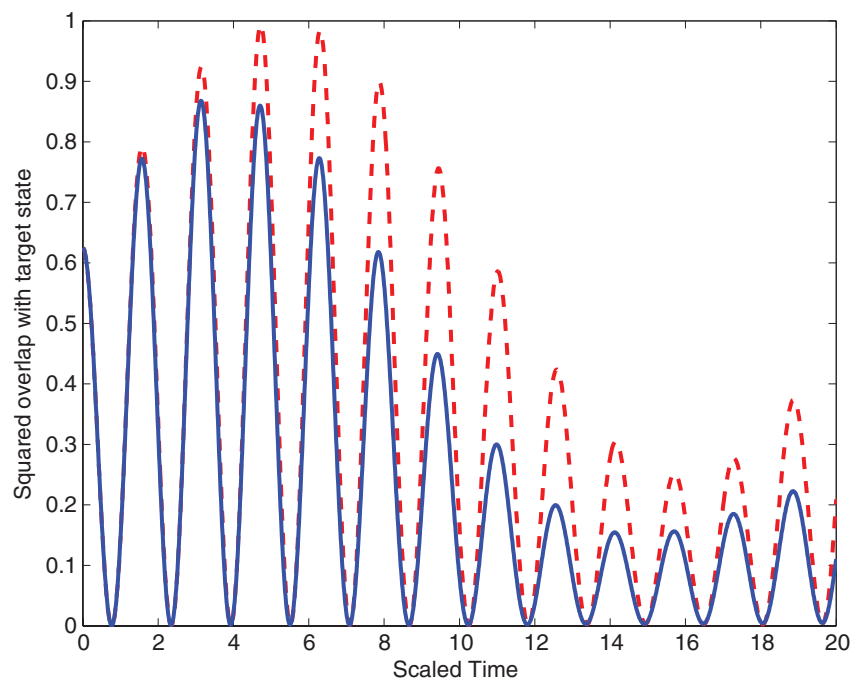

FIG. 5. (Color online) This plot shows the effects of decoherence on an initial state $\frac{1}{\sqrt{2}}\left(|\uparrow\rangle_{A}|\uparrow\rangle_{B}+|\uparrow\rangle_{A}|\downarrow\rangle_{B}\right)$. It plots the evolution of the squared overlap of this state with its intended target state at the end of the gate, namely, $\frac{1}{\sqrt{2}}|\uparrow\rangle_{A}|\uparrow\rangle_{B}+\frac{e^{i \pi / 4}}{2}\left(|\uparrow\rangle_{A}|\downarrow\rangle_{B}-i|\downarrow\rangle_{A}|\uparrow\rangle_{B}\right)$. The dashed curve shows the evolution when only charge decoherence is present, while the solid curve presents the evolution when both the charge as well as hyperfine induced decoherences are present. Charge noise is set so as to have a charge decoherence time scale of about $1 \mathrm{~ns}$, while the strength of the random nuclear field causing the spin decoherence is set to the realistic value of $B_{\text {nuc }} \sim 0.1$ in scaled units (with $10 \mu \mathrm{eV}$ taken as unity). The parameters used in the plot are $t=\sqrt{2}$ and $U=20$ in scaled units where the energy scale $10 \mu \mathrm{eV}$ is set to unity (one unit of the scaled time is about $0.45 \mathrm{~ns}$ ). have performed some numerics to check that the error is not significant for small deviations from this tuning. For example, for a $1 \%$ difference between $t_{\mathrm{AC}}$ and $t_{\mathrm{CB}}$, we have about a $0.7 \%$ change in the relevant squared overlaps at a given instant of time. Similarly, for a $1 \%$ difference of $U_{\mathrm{C}}$ from $U_{\mathrm{A}}$ and $U_{\mathrm{B}}$, we have about a $1.5 \%$ change in the relevant squared overlaps at a given instant of time.

\section{GATES IN A HIGH DECOHERENCE REGIME}

Suppose one has a very high charge decoherence (so that coherence stays, say, for only $0.1 \mathrm{~ns}$ ) then one can still use our triple-dot setup for a gate by stopping at the very first peak of the oscillation of the $|\uparrow\rangle_{A}|\uparrow\rangle_{B}$ state, i.e., at a time $\tau_{1}=2 \pi / t \sim 0.1 \mathrm{~ns}$. The resulting quantum gate is, however, different and obtained by replacing the right-hand sides of the second and third rows of Eq. (2) (in the $t \gg J$ limit) by $e^{i 3 J \tau / 2}\left(\cos 3 J \tau / 2|\uparrow\rangle_{A}|\downarrow\rangle_{B}-i \sin 3 J \tau / 2|\downarrow\rangle_{A}|\uparrow\rangle_{B}\right)$ and $e^{i 3 J \tau / 2}\left(\cos 3 J \tau / 2|\downarrow\rangle_{A}|\uparrow\rangle_{B}-i \sin 3 J \tau / 2|\uparrow\rangle_{A}|\downarrow\rangle_{B}\right)$, respectively. This has a lower entangling power, but is nonetheless an entangling gate, still useful for universal quantum computation. One merely has to halt the Hamiltonian at an earlier time (before decoherence has become too prominent) to get the gate and repeat the gate a few times to get a maximally entangling gate such as a CNOT from it. In Fig. 6, we have plotted the overlap of the ideal target state $e^{i 3 J \tau / 2}\left(\cos 3 J \tau / 2|\uparrow\rangle_{A}|\downarrow\rangle_{B}-i \sin 3 J \tau / 2|\downarrow\rangle_{A}|\uparrow\rangle_{B}\right)$ when one

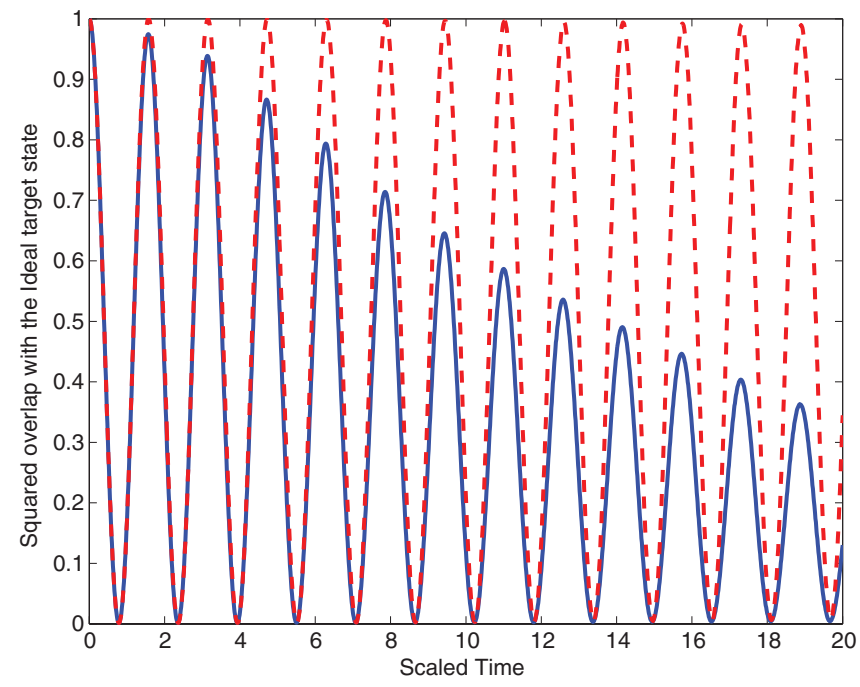

FIG. 6. (Color online) This plot shows the effects of decoherence on an initial state $|\uparrow\rangle_{A}|\downarrow\rangle_{B}$. It plots the evolution of the squared overlap of this state, under both mechanisms of decoherence, with the state that it evolves to at any time $\tau$ under ideal conditions (i.e., $t \ll U$ and no decoherence), namely $e^{i 3 J \tau / 2}\left(\cos 3 J \tau / 2|\uparrow\rangle_{A}|\downarrow\rangle_{B}-\right.$ $\left.i \sin 3 J \tau / 2|\downarrow\rangle_{A}|\uparrow\rangle_{B}\right)$. The dashed curve shows the evolution when only charge decoherence is present, while the solid curve presents the evolution when both the charge as well as hyperfine induced decoherences are present. Charge noise is set so as to have a charge decoherence time scale of about $1 \mathrm{~ns}$, while the strength of the random nuclear field causing the spin decoherence is set to the realistic value of $B_{\text {nuc }} \sim 0.1$ in scaled units (with $10 \mu \mathrm{eV}$ taken as unity). The parameters used in the plot are $t=\sqrt{2}$ and $U=20$ in scaled units (one unit of the scaled time is about $0.45 \mathrm{~ns}$ ). 
starts from the state $|\uparrow\rangle_{A}|\downarrow\rangle_{B}$ and has an evolution under the presence of both mechanisms of decoherence.

\section{DISCUSSIONS}

The primary achievement in this paper is to show that using triple-dot systems, one can encode two single spin qubits and have an entangling quantum gate (more precisely a $\sqrt{\mathrm{SWAP}}$ gate) between them merely by tuning the voltage of the central dot (or voltage misalignment between the dots). This eases the restriction of having to tune the tunnel coupling $t$ on a fast time scale, which might be difficult ${ }^{4}$ or even impossible to tune in some setups of permanently built dots. One can scale this scheme to several qubits by using a one-dimensional array in a $A B A B A B \ldots A B A$ scenario with the $A$ sites having single qubits and the $B$ sites being empty in the nonoperative state of the system. Whenever a quantum gate between two qubits is required, we tune the voltage of only the $B$ site between the qubits to enable a gate between them. We have shown that the gate works with high enough fidelities for a variety of input states for achievable values of charge and spin decoherence rates. For stronger charge decoherence, one can halt the unitary evolution at earlier pertinent times and still get an entangling gate, albeit with lower power.

To summarize, before concluding, let us clarify the physical mechanism for the gates found by us. As there is a high $U$, only single electrons can occupy each dot. Starting from the initial $(1,0,1)$ charge configuration, dynamics evolves the electrons to both $(1,1,0)$ and $(0,1,1)$ configurations due to the tunneling $t$ and this is indeed the time scale for the fastest oscillations seen in the time evolution of states. While residing in the
$(1,1,0)$ and $(0,1,1)$ configurations (the amplitude for which is itself evolving with time), the spins of the neighboring dots interact via exchange coupling $J$. Thus, on the slower time scale of $J$, our entangling gates are implemented. A nontrivial fact in the analysis was that the empty site prohibited one from labeling the electrons by their sites so that it was convenient for us to use a second quantized treatment. However, the most surprising outcome was that we were able to find special times at which both a maximally entangling gate was implemented as well as the electrons returned back to their original sites $\mathrm{A}$ and $\mathrm{B}$. This enabled us to state that a quantum gate has occurred between the spin degree of freedom of qubits $\mathrm{A}$ and $\mathrm{B}$, though at intermediate times, when one electron was at $\mathrm{C}$, one of the qubits, either qubit A or qubit $\mathrm{B}$, would have been impossible to define.

\section{ACKNOWLEDGMENTS}

S.B. acknowledges the support of the ERC and the UK Engineering and Physical Sciences Research Council (EPSRC). When most of this work was done, S.B. was also supported by the Royal Society and the Wolfson Foundation and J.G.C. was supported by the EPSRC sponsored Quantum Information Processing Interdisciplinary Research Centre (QIPIRC). We have benetted from discussions with John Jefferson, Brendon Lovett, Simon Benjamin and Andrew Briggs during early stages of this work. Additionally, during a visit to the Institute for Theoretical Atomic, Molecular and Optical Physics (ITAMP), Harvard, in 2008, S.B. benetted from some discussions with M. D. Lukin, J. M. Taylor and C. M. Marcus during the formative stages of this work. *j.coello@ucl.ac.uk

${ }^{1}$ D. Loss and D. P. DiVincenzo, Phys. Rev. A 57, 120 (1998).

${ }^{2}$ J. R. Petta, A. C. Johnson, J. M. Taylor, E. A. Laird, A. Yacoby, M. D. Lukin, C. M. Marcus, M. P. Hanson, and A. C. Gossard, Science 309, 2180 (2005).

${ }^{3}$ R. Hanson, L. H. Willems vanBeveren, I. T. Vink, J. M. Elzerman, W. J. M. Naber, F. H. L. Koppens, L. P. Kouwenhoven, and L. M. K. Vandersypen, Phys. Rev. Lett. 94, 196802 (2005).

${ }^{4}$ R. Hanson and G. Burkard, Phys. Rev. Lett. 98, 050502 (2007).

${ }^{5}$ H. A. Engel, L. P. Kouwenhoven, D. Loss, and C. M. Marcus, Quant. Info. Proc. 3, 115 (2004).

${ }^{6}$ J. M. Taylor, J. R. Petta, A. C. Johnson, A. Yacoby, C. M. Marcus, and M. D. Lukin, Phys. Rev. B 76, 035315 (2007).

${ }^{7}$ J. H. Jefferson, M. Fearn, D. L. J. Tipton, and T. P. Spiller, Phys. Rev. A 66, 042328 (2002).

${ }^{8}$ G. Ortner, M. Bayer, Y. Lyanda-Geller, T. L. Reinecke, A. Kress, J. P. Reithmaier, and A. Forchel, Phys. Rev. Lett. 94, 157401 (2005).

${ }^{9}$ C. Flindt, A. S. Sorensen, M. D. Lukin, and J. M. Taylor, Phys. Rev. Lett. 98, 240501 (2007).

${ }^{10}$ D. Schroer, A. D. Greentree, L. Gaudreau, K. Eberl, L. C. L. Hollenberg, J. P. Kotthaus, and S. Ludwig, Phys. Rev. B 76, 075306 (2007); G. Yamahata, Yoshishige Tsuchiya, Hiroshi Mizuta,
K. Uchida, and S. Oda, Solid-State Electronics 53, 779 (2009); M. Pierre, R. Wacquez, B. Roche1, X. Jehl, M. Sanquer, M. Vinet, E. Prati, M. Belli, and M. Fanciulli, Appl. Phys. Lett. 95, 242107 (2009).

${ }^{11}$ E. A. Laird, J. M. Taylor, D. P. DiVincenzo, C. M. Marcus, M. P. Hanson, and A. C. Gossard, Phys. Rev. B 82, 075403 (2010).

${ }^{12}$ J. Lehmann, A. Gaita-Ariño, E. Coronado, and D. Loss, Nat. Nanotechnology 2, 312 (2007).

${ }^{13}$ D. P. DiVincenzo, D. Bacon, J. Kempe, G. Burkard, and K. B. Whaley, Nature (London) 408, 339 (2000).

${ }^{14}$ D. Bacon, K. R. Brown, and K. B. Whaley, Phys. Rev. Lett. 87, 247902 (2001); Y. S. Weinstein and C. S. Hellberg, ibid. 98, 110501 (2007).

${ }^{15}$ C. S. Hellberg, arXiv:quant-ph/0304150.

${ }^{16}$ J. P. Kestner and S. Das Sarma, Phys. Rev. A 84, 012315 (2011).

${ }^{17}$ G. Giavaras, J. H. Jefferson, M. Fearn, and C. J. Lambert, Phys. Rev. B 75, 085302 (2007).

${ }^{18}$ J. G. Coello, A. Bayat, S. Bose, J. H. Jefferson, and C. E. Creffield, Phys. Rev. Lett. 105, 080502 (2010).

${ }^{19}$ C. A. Stafford and S. Das Sarma, Phys. Rev. Lett. 72, 3590 (1994); R. Kotlyar, C. A. Stafford, and S. Das Sarma, Phys. Rev. B 58, R1746 (1998); C. A. Stafford, R. Kotlyar, and S. Das Sarma, ibid. 
58, 7091 (1998); M. R. Wegewijs and Y. V. Nazarov, ibid. 60, 14318 (1999).

${ }^{20}$ M. J. Bremner, C. M. Dawson, J. L. Dodd, A. Gilchrist, A. W. Harrow, D. Mortimer, M. A. Nielsen, and T. J. Osborne, Phys. Rev. Lett. 89, 247902 (2002).

${ }^{21}$ L. Gaudreau, S. A. Studenikin, A. S. Sachrajda, P. Zawadzki, A. Kam, J. Lapointe, M. Korkusinski, and P. Hawrylak, Phys. Rev. Lett. 97, 036807 (2006).

${ }^{22}$ T. Byrnes, N. Y. Kim, K. Kusudo, and Y. Yamamoto, Phys. Rev. B 78, 075320 (2008).
${ }^{23}$ G. Shinkai, T. Hayashi, T. Ota, and T. Fujisawa, Phys. Rev. Lett. 103, 056802 (2009).

${ }^{24}$ J. Gorman, D. G. Hasko, and D. A. Williams, Phys. Rev. Lett. 95, 090502 (2005).

${ }^{25}$ D. J. Reilly, J. M. Taylor, J. R. Petta, C. M. Marcus, M. P. Hanson, and A. C. Gossard, Science 321, 781 (2008).

${ }^{26}$ S. Trotzky, P. Cheinet, S. Flling, M. Feld, U. Schnorrberger, A. M. Rey, A. Polkovnikov, E. A. Demler, M. D. Lukin, and I. Bloch, Science 319, 295 (2008). 